\title{
The Meditative Mind: A Comprehensive Meta-Analysis of MRI Studies
}

\author{
Maddalena Boccia, ${ }^{1,2}$ Laura Piccardi, ${ }^{2,3}$ and Paola Guariglia ${ }^{4}$ \\ ${ }^{1}$ Department of Psychology, "Sapienza” University of Rome, Via dei Marsi 78, 00185 Rome, Italy \\ ${ }^{2}$ Neuropsychology Unit, IRCCS Fondazione Santa Lucia, Via Ardeatina 306, 00179 Rome, Italy \\ ${ }^{3}$ Department of Life, Health and Environmental Sciences, L' Aquila University, P.le S. Tommasi 1, 67100 Coppito, Italy \\ ${ }^{4}$ Department of Human Science and Society, University of Enna "Kore," Cittadella Universitaria, 94100 Enna, Italy \\ Correspondence should be addressed to Maddalena Boccia; maddalena.boccia@gmail.com
}

Received 25 August 2014; Accepted 16 November 2014

Academic Editor: Patricia Gerbarg

Copyright ( 2015 Maddalena Boccia et al. This is an open access article distributed under the Creative Commons Attribution License, which permits unrestricted use, distribution, and reproduction in any medium, provided the original work is properly cited.

Over the past decade mind and body practices, such as yoga and meditation, have raised interest in different scientific fields; in particular, the physiological mechanisms underlying the beneficial effects observed in meditators have been investigated. Neuroimaging studies have studied the effects of meditation on brain structure and function and findings have helped clarify the biological underpinnings of the positive effects of meditation practice and the possible integration of this technique in standard therapy. The large amount of data collected thus far allows drawing some conclusions about the neural effects of meditation practice. In the present study we used activation likelihood estimation (ALE) analysis to make a coordinate-based meta-analysis of neuroimaging data on the effects of meditation on brain structure and function. Results indicate that meditation leads to activation in brain areas involved in processing self-relevant information, self-regulation, focused problem-solving, adaptive behavior, and interoception. Results also show that meditation practice induces functional and structural brain modifications in expert meditators, especially in areas involved in self-referential processes such as self-awareness and self-regulation. These results demonstrate that a biological substrate underlies the positive pervasive effect of meditation practice and suggest that meditation techniques could be adopted in clinical populations and to prevent disease.

\section{Introduction}

Mind and body practices such as yoga, meditation, progressive relaxation, or guided imagery use mental and physical abilities to improve health and well-being. Over the past decade these practices have received increasing attention in different fields of study in which the physiological mechanisms underlying the beneficial effects observed in trained individuals have been investigated. Increased knowledge about the physiological effects of mind and body practices makes it possible to explore their therapeutic potential, identify adverse effects, and safely integrate these techniques into standard therapeutic approach.

Meditation is a complex process aimed at self-regulating the body and mind and is often associated with psychological and neurophysiological modifications [1]. Meditation practices can be oriented toward the concentration of attention on a particular external, corporal, or mental object, while ignoring all irrelevant stimuli (focused attention meditation), or toward techniques that try to enlarge the attentional focus to all incoming sensations, emotions, and thoughts from moment to moment without focusing on any of them (open monitoring meditation) [2]. In any case, most meditation approaches use both types of practices complementarily [3, 4].

Meditation practice has been found to promote wellbeing by fostering cognitive and emotional processes $[5,6]$. Specifically, it has been found to improve working memory and attentional processes [7-9] as well as perceptual abilities [10]. It has also been found to promote prosocial behavior [11] and emotional regulation [12]. The potential contribution of meditation to cognitive and emotional processes can be appreciated in the context of the model proposed by Lutz and colleagues $[13,14]$. These authors posited that meditation 
practice induces enhancement of at least four different abilities: sustained attention, monitoring faculty (to detect mind wandering), the ability to disengage from a distracting object without further involvement (attentional switching), and the ability to redirect focus to the chosen object (selective attention). A recent systematic review by Chiesa and colleagues [15] allowed drawing some important conclusions about the positive effect of meditation on cognitive functions. Executive functions, attention, and memory were the main targets of meditation practice. In particular, as compared to the control group, meditators showed improved sustained attention [16], conflict monitoring [7], and reduced attentional blink [17]. Meditators also performed better than controls in the classical working memory paradigms $[16,18]$. Concerning memory, significant improvement was found in meta-awareness [19] and in specific autobiographical memories [20] after meditation training. Ortner and colleagues [21] also found that meditation groups showed reduced interference from unpleasant pictures, suggesting that meditation also has a positive effect in decreasing emotional interference during performance of a cognitive task.

Interestingly, the current literature suggests that meditation has a potential effect on age-related cognitive decline [22, 23], probably due to the regulation of glucocorticosteroids, inflammation, and serotonin metabolism [23]. Furthermore, it has been hypothesized [24] that the stress reduction promoted by meditation contrasts hippocampal vulnerability to neurotoxicity [25] and leads to increased hippocampal grey matter volume due to neuron preservation and/or neurogenesis. Meditation has also been found to reduce a number of psychological and physical symptoms in clinical populations [26, 27]. King and coworkers [28] found that mindfulness-based cognitive therapy was an acceptable brief intervention therapy for combatting PTSD: indeed, it reduced avoidance symptoms and PTSD cognitions. There is also evidence that, compared to standard care, mindfulness-based cognitive therapy almost halves the risk of relapse in people who are currently well but who have experienced at least three prior episodes of depression $[29,30]$ and is comparable to antidepressant medication in reducing risk of relapse [31].

The effects of meditation on brain structure and function have received increasing attention in neuroimaging studies (MRI, fMRI, and PET) and the number of published studies is steadily growing [32]. Specifically, the findings of neuroimaging investigations have allowed linking the positive effects of meditation to specific brain modifications. Neuroimaging studies of brain modification can be roughly divided into those investigating (1) neurofunctional correlates of meditation, (2) neurofunctional modifications after meditation training, and (3) structural brain modifications in expert meditators.

Functional studies on the brain correlates of meditation have assessed neural activation during meditation by requiring participants to undergo fMRI scans during meditation tasks. These studies have reported increased activation in areas associated with attention, mind wandering, retrieval of episodic memories, and emotional processing during meditation [33]. Specifically, increased activation in the prefrontal cortex [34], parietal areas [35], middle cingulate cortex, and hippocampal and parahippocampal formations [36] has been reported.

Studies of functional brain modifications after meditation training have focused on functional and the metabolic changes after meditation training and/or in expert meditators compared with control participants. These studies adopted different paradigms (Table 1): the affective Stroop task [37], pain-related tasks [38-41], attentional paradigms [42-44], emotional provocation [45], and meditation tasks [36, 46, 47]. The results of these studies are very intriguing because they shed more light on the possible link between neurofunctional changes and the positive effect of meditation on different aspects of cognitive and emotional processes, such as perceptual and attentional processes [7-10] as well as social behavior [11] and emotional regulation [12].

The studies that investigated structural brain modifications in expert meditators (Table 2) focused on brain structural changes after meditation training and/or in expert meditators compared with control participants; they primarily assessed grey matter changes with whole-brain voxelbased morphometry or cortical thickness mapping of MRI data [24, 48-54]. These studies principally found that, compared with control participants, expert meditators showed increased grey matter volume at the level of the posterior cingulate cortex, temporoparietal junction, angular gyrus, orbitofrontal cortex, hippocampus, and subiculum in the medial temporal lobe and the brainstem.

Previous neuroimaging studies on the effects of meditation on brain structure and function adopted different meditation techniques and recruited participants with different meditation training. For example, some studies recruited Buddhist practitioners [44] and others recruited participants with experience in SOHAM meditation [47] or ACEM meditation [33]. Several studies reported that different meditation techniques require different cognitive processes and thus produce different neural effects $[55,56]$. But, despite differences in meditation techniques and underlying cognitive processes, it has been proposed that all meditation techniques share a central process that supports their common goal, that is, inducing relaxation, regulating attention, and developing an attitude of detachment from one's own thoughts [57]. Evidence from a recent meta-analysis of ten neuroimaging studies [57] seems to suggest that the caudate body, entorhinal cortex, and medial prefrontal cortex have a central role in supporting the general aspects of meditation effects.

The large amount of data collected over the past decade allows drawing some definite conclusions about the neural effects of meditation practice and allows discussing the positive effects of meditation practice from a biological point of view.

The main aim of the present study was to draw some definite conclusions about the neural network activated during meditation tasks and to explore functional (fMRI) and structural (sMRI) changes in expert meditators. To pursue this aim we adopted a meta-analytic approach based on activation likelihood estimation (ALE) analysis, which allows performing coordinate-based meta-analyses of neuroimaging data [58]. 
TABLE 1: Functional changes in meditators.

\begin{tabular}{|c|c|c|c|c|}
\hline Paper & $N$ & Contrast & Experience & Meditation \\
\hline Allen et al., 2012 [37] & 61 & AFT, task > passive view & 6 weeks & MT \\
\hline Allen et al., 2012 [37] & 61 & AFT, negative $>$ neutral & 6 weeks & MT \\
\hline Allen et al., 2012 [37] & 61 & AFT, task by emotion & 6 weeks & MT \\
\hline $\begin{array}{l}\text { Brefczynski-Lewis et al., } \\
2007 \text { [44] }\end{array}$ & 41 & $\mathrm{EM}>\mathrm{NM}$ during meditation & - & Buddhist practitioners \\
\hline $\begin{array}{l}\text { Brefczynski-Lewis et al., } \\
2007 \text { [44] }\end{array}$ & 41 & $\mathrm{EM}>\mathrm{INM}$ during meditation & - & Buddhist practitioners \\
\hline $\begin{array}{l}\text { Brefczynski-Lewis et al., } \\
2007 \text { [44] }\end{array}$ & 41 & $\mathrm{EM}>\mathrm{INM}$ meditation $>$ rest, group by task & - & Buddhist practitioners \\
\hline Creswell et al., 2007 [86] & 27 & Neural areas associated with MAAS & - & - \\
\hline Davanger et al., 2010 [34] & 4 & ACEM meditation > control task & 23 years & ACEM \\
\hline Ding et al., 2014 [87] & 32 & IBMT > RT & 10 days & IBMT \\
\hline Engström et al., 2010 [36] & 8 & Meditate & 14 months & ACEM and Kundalini \\
\hline Engström et al., 2010 [36] & 8 & Word & 14 months & ACEM and Kundalini \\
\hline Engström et al., 2010 [36] & 8 & Silent mantra & 14 months & ACEM and Kundalini \\
\hline Farb et al., 2007 [42] & 27 & Experiential focus, MT $>$ controls & 8 weeks & MBSR \\
\hline Farb et al., 2010 [88] & 36 & Sadness provocation, $\mathrm{MT}>$ controls & 8 weeks & MBSR \\
\hline Farb et al., 2013 [89] & 36 & Interoception $>$ exteroception, $\mathrm{MT}>$ controls & 8 weeks & MBSR \\
\hline Grant et al., 2011 [38] & 22 & Pain, EM > controls & - & Zen \\
\hline Grant et al., 2011 [38] & 22 & Hot $>$ warm, EM & - & Zen \\
\hline Grant et al., $2011[38]$ & 22 & Pain, EM > controls & - & Zen \\
\hline Guleria et al., 2013 [47] & 14 & Meditation $>$ control & $5.8 \pm 0.9$ years & SOHAM \\
\hline $\begin{array}{l}\text { Hasenkamp et al., } 2012 \\
{[43]}\end{array}$ & 14 & AWARE-MW & $>1$ year & FAM \\
\hline $\begin{array}{l}\text { Hasenkamp et al., } 2012 \\
\text { [43] }\end{array}$ & 14 & SHIFT > MW & $>1$ year & FAM \\
\hline $\begin{array}{l}\text { Hasenkamp et al., } 2012 \\
\text { [43] }\end{array}$ & 14 & FOCUS $>$ MW & $>1$ year & FAM \\
\hline $\begin{array}{l}\text { Hasenkamp et al., } 2012 \\
\text { [43] }\end{array}$ & 14 & $\mathrm{MW}>\mathrm{SHIFT}$ & $>1$ year & FAM \\
\hline $\begin{array}{l}\text { Hasenkamp et al., } 2012 \\
\text { [43] }\end{array}$ & 14 & Correlations with practice time, AWARE & $>1$ year & FAM \\
\hline $\begin{array}{l}\text { Hasenkamp et al., } 2012 \\
\text { [43] }\end{array}$ & 14 & Correlations with practice time, SHIFT & $>1$ year & FAM \\
\hline $\begin{array}{l}\text { Hasenkamp et al., } 2012 \\
\text { [43] }\end{array}$ & 14 & Correlations with practice time, FOCUS & $>1$ year & FAM \\
\hline Hölzel et al., 2007 [12] & 30 & Mindfulness $>$ arithmetic, EM & $>2$ years & Vipassana \\
\hline Hölzel et al., 2007 [12] & 30 & $\mathrm{EM}>$ controls & $>2$ years & Vipassana \\
\hline $\begin{array}{l}\text { Ives-Deliperi et al., } 2011 \\
{[90]}\end{array}$ & 10 & Mindfulness > control in EM & 8 weeks & MBSR \\
\hline Jang et al., 2011 [91] & 68 & $\mathrm{EM}>$ controls & $\begin{array}{l}39.88 \pm 25.58 \\
\text { months }\end{array}$ & BWVM \\
\hline Kilpatrick et al., 2011 [92] & 32 & Auditory/salience & 8 weeks & MBSR \\
\hline Kilpatrick et al., 2011 [92] & 32 & Medial visual & 8 weeks & MBSR \\
\hline Kilpatrick et al., 2011 [92] & 32 & Lateral visual & 8 weeks & MBSR \\
\hline Kilpatrick et al., 2011 [92] & 32 & Sensorimotor & 8 weeks & MBSR \\
\hline Kilpatrick et al., 2011 [92] & 32 & Executive control & 8 weeks & MBSR \\
\hline Lee et al., 2012 [93] & 44 & $\mathrm{CPT}$ in $\mathrm{FAM}$ & $>5$ years & FAM/LKM \\
\hline Lee et al., 2012 [93] & 44 & EPT-happy in FAM & $>5$ years & FAM/LKM \\
\hline Lee et al., 2012 [93] & 44 & EPT-happy in LKM & $>5$ years & FAM/LKM \\
\hline
\end{tabular}


TABle 1: Continued.

\begin{tabular}{|c|c|c|c|c|}
\hline Paper & $N$ & Contrast & Experience & Meditation \\
\hline Lee et al., 2012 [93] & 44 & EPT-sad in FAM & $>5$ years & FAM/LKM \\
\hline Lee et al., 2012 [93] & 44 & EPT-sad in LKM & $>5$ years & FAM/LKM \\
\hline Lutz et al., 2008 [14] & 28 & Meditation $>$ resting states, EM > controls & $\begin{array}{l}10000 \text { to } 50000 \\
\text { hours }\end{array}$ & Buddhist practitioners \\
\hline Lutz et al., 2009 [94] & 22 & Meditation > resting states, EM > controls & $\begin{array}{l}10000 \text { to } 50000 \\
\text { hours }\end{array}$ & Buddhist practitioners \\
\hline Lutz et al., 2013 [39] & 28 & Hot $>$ warm, EM $>$ controls & $>10000$ hours & Buddhist practitioners \\
\hline Lutz et al., 2013 [39] & 28 & $\mathrm{EM}>$ controls & $>10000$ hours & Buddhist practitioners \\
\hline Manna et al., 2010 [56] & 8 & FAM > rest, in EM & $\begin{array}{l}\text { Mean } 15750 \\
\text { hours }\end{array}$ & Buddhist monks \\
\hline Manna et al., 2010 [56] & 8 & $\mathrm{OM}>\mathrm{FAM}$, in EM & $\begin{array}{l}\text { Mean } 15750 \\
\text { hours }\end{array}$ & Buddhist monks \\
\hline Manna et al., 2010 [56] & 8 & $\mathrm{OM}>$ rest, in EM & $\begin{array}{l}\text { Mean } 15750 \\
\text { hours }\end{array}$ & Buddhist monks \\
\hline Mascaro et al., 2013a [40] & 29 & Self pain task, pain $>$ no pain & 8 weeks & $\mathrm{CBCT}$ \\
\hline Mascaro et al., 2013a [40] & 29 & Other pain tasks, pain $>$ no pain & 8 weeks & $\mathrm{CBCT}$ \\
\hline Mascaro et al., 2013b [41] & 29 & RME, emotion $>$ gender & 8 weeks & СВСТ \\
\hline Monti et al., 2012 [95] & 8 & Post- > pretreatment & 8 weeks & MBAT \\
\hline Monti et al., 2012 [95] & 8 & Post- $>$ pretreatment, MBAT $>$ controls & 8 weeks & MBAT \\
\hline $\begin{array}{l}\text { Orme-Johnson et al., } 2006 \\
\text { [96] }\end{array}$ & 24 & Post- > pretreatment & $31.3 \pm 2.3$ years & TMT \\
\hline Taylor et al., 2011 [45] & 22 & Positive $>$ neutral pictures & 1000 hours & Zen \\
\hline Tang et al., 2013 [46] & 60 & $\mathrm{IBMT}>\mathrm{RT}$ & 10 sessions & IBMT \\
\hline Tang et al., 2013 [46] & 60 & IBMT, post > pre & 10 sessions & IBMT \\
\hline Wang et al., 2011 [97] & 10 & Meditation $1>$ control & 30 years & Kundalini \\
\hline Wang et al., 2011 [97] & 10 & Meditation $2>$ control & 30 years & Kundalini \\
\hline Wang et al., 2011 [97] & 10 & Meditation $2>$ Meditation 1 & 30 years & Kundalini \\
\hline Wang et al., 2011 [97] & 10 & Baseline $2>$ Baseline 1 & 30 years & Kundalini \\
\hline Xu et al., 2014 [33] & 14 & $\mathrm{NDM}>$ rest & $27 \pm 9$ years & ACEM \\
\hline Xu et al., 2014 [33] & 14 & Concentrative practicing $>$ rest & $27 \pm 9$ years & ACEM \\
\hline Xu et al., 2014 [33] & 14 & NDM $>$ concentrative practicing $>$ rest & $27 \pm 9$ years & ACEM \\
\hline
\end{tabular}

Notes. AST: affective Stroop task; MT: mindfulness training; EM: expert meditators; NM: novice meditators; INM: incentive novice meditators; MAAS: Mindful Attention Awareness Scale; IBMT: integrative body-mind training; RT: relaxation training; MBSR: mindfulness-based stress reduction; BWVM: brain-wave vibration meditation; FAM: focused attention meditation; LKM: loving-kindness meditation; CPT: continuous performance test; EPT: emotion-processing task; OM: open monitoring meditation; СBCT: cognitively based compassion training; RME: reading the mind eyes test; MBAT: mindfulness-based art therapy; TMT: transcendental meditation technique; NDM: nondirective meditation; MW: mind wondering.

TABLE 2: sMRI studies on expert meditators.

\begin{tabular}{lcccc}
\hline Paper & $N$ & Contrast & Experience & Meditation \\
\hline Kang et al., 2013 [49] & 92 & Meditators versus controls & $41.23 \pm 27.57$ months & BWV \\
Wei et al., 2013 [54] & 40 & Meditators versus controls & $14 \pm 8$ years & TCC \\
Hölzel et al., 2011 [48] & 16 & Pre- to post-MBSR training & 8 weeks & MBSR \\
Kurth et al., 2014 [50] & 100 & Meditators versus controls & $19.8 \pm 11.4$ years & - \\
Kurth et al., 2014 [50] & 100 & Correlation with meditation practice & $19.8 \pm 11.4$ years & - \\
Leung et al., 2013 [51] & 25 & Meditators versus controls & years & LKM \\
Luders et al., 2009 [6, 52] & 44 & Meditators versus controls & $24.18 \pm 12.36$ years & - \\
Luders et al., 2009 [6, 52] & 44 & Meditators versus controls & $24.18 \pm 12.36$ years & $19.8 \pm 11.4$ years \\
Luders et al., 2013 [24] & 100 & Meditators versus controls & $16.5 \pm 5.1$ years & Tibetan Buddhism \\
Vestergaard-Poulsen et al., 2009 [53] & 20 & Meditators versus controls & -
\end{tabular}

Notes. BWV: brain-wave vibration; TCC: Tai Chi Chuan; MBSR: mindfulness-based stress reduction; LKM: loving-kindness meditation. 


\section{Method}

2.1. Inclusion Criteria for Papers. The database search on PubMed was performed using the following string: (((()(MRI) AND meditation) NOT Alzheimer's) NOT Parkinson's) NOT EEG) NOT MEG) NOT mild cognitive impairment). A total of 93 papers emerged. From this collection, we selected only papers that (1) included wholebrain analysis performed using magnetic resonance imaging (MRI), (2) provided coordinates of activation foci either in Montreal Neurological Institute (MNI) or in Talairach reference space, (3) studied young and healthy participants, (4) reported activation from group studies, (5) included meditators or required participants to perform a meditation task, and (6) used no pharmacological manipulation. We selected 57 papers: 42 reported fMRI studies and 15, sMRI studies. Out of the $42 \mathrm{fMRI}$ studies, 5 were excluded because they did not provide coordinates of activation foci; out of the 15 papers on sMRI studies, 6 papers were excluded for the same reason and one paper was excluded because it did not include expert meditators.

In line with the aims of the present meta-analysis, individual experimental studies from selected papers were divided according to three main axes: papers reporting (a) functional magnetic resonance imaging studies (fMRI) during meditation training, (b) functional magnetic resonance imaging studies (fMRI) that studied neural modifications after meditation training, and (c) structural MRI studies (sMRI). Note that the fMRI studies on neural modifications after meditation (see Table 1) included those that adopted different paradigms. These studies also reported the results of comparisons between pre- and posttreatment or results of comparisons between expert meditators and naïve participants. A meta-analytic approach, which models the probability distributions centered at the coordinates of each activation focus, allows obtaining a general picture of functional neural modifications in meditators.

We included 37 individual fMRI experimental studies on functional activations during meditation tasks (642 participants), $63 \mathrm{fMRI}$ experimental studies (see Table 1 for more details) on functional changes ascribable to meditation (1,652 participants including both meditators and controls), and 10 experimental sMRI studies (Table 2) on structural changes ascribable to meditation (581 participants).

2.2. Activation Likelihood Estimation (ALE). Activation likelihood estimation (ALE) analyzes the probability that a voxel will contain at least one of the activation foci; it is calculated at each voxel and results in a thresholded ALE map. In other words, ALE assesses the overlap between foci by modeling the probability distributions centered at the coordinates of each one [58].

Our first aim was to provide a general picture of areas activated during meditation tasks. Thus, we carried out an ALE analysis of fMRI studies on functional activations during meditation tasks. Then, we performed two ALE analyses to determine whether meditation produces consistent modifications in brain structure and function. In the first analysis we included sMRI studies, and in the second analysis we included fMRI studies on neural modifications after meditation training.

The ALE meta-analysis was performed using GingerALE 2.1.1 (http://brainmap.org/) with MNI coordinates (Talairach coordinates were automatically converted into MNI coordinates by GingerALE.). Following Eickhoff et al's modified procedure [58], the ALE values of each voxel in the brain were computed and a test was performed to determine the null distribution of the ALE statistic of each voxel. The FWHM value was automatically computed because this parameter is empirically determined [58].

For the fMRI studies, the thresholded ALE map was computed using $P$ values from the previous step and a false discovery rate (FDR) at the 0.05 level of significance (Tom Nichol's FDR algorithm). Moreover, a minimum cluster size of $200 \mathrm{~mm}^{3}$ was chosen. A cluster analysis was performed on the thresholded map.

For the sMRI studies, the thresholded ALE map was computed using $P$ values from the previous step and a cluster level correction at the 0.05 level of significance, with a minimum cluster size of $200 \mathrm{~mm}^{3}$. A cluster analysis was performed on the thresholded map.

The ALE results were registered on an MNI-normalized template (http://brainmap.org/) using Mricro (http://www .mccauslandcenter.sc.edu/mricro/index.html).

\section{Results}

3.1. Brain Areas Activated during Meditation Tasks. ALE meta-analysis of fMRI studies carried out during meditation revealed a network of areas spanning from the occipital to the frontal lobes that was more highly activated during the meditation condition than the control condition. This network included the caudate nuclei and insula bilaterally, the precuneus, middle and superior temporal gyrus, and precentral gyrus in the left hemisphere, and the anterior cingulate cortex, superior frontal gyrus, parahippocampal gyrus, inferior parietal lobule (angular gyrus), and middle occipital gyrus in the right hemisphere. We also found that left posterior cerebellum, specifically the declive, was more highly activated during meditation than the control condition (Figure 1).

3.2. Functional Modifications in Meditators. We found that meditation practice (see Table 1) was associated with increased functional activation in a wide network of areas including the bilateral middle frontal gyrus, precentral gyrus, anterior cingulate cortex, insula, and claustrum. In the left hemisphere we also found increased activation at the level of the inferior frontal gyrus, precuneus, caudate nucleus, and thalamus; and in the right hemisphere we found increased activation in the medial frontal gyrus, parahippocampal gyrus, middle occipital gyrus, inferior parietal lobule, and lentiform nucleus (Figure 2).

3.3. Structural Modifications in Meditators. We found that meditation practice was associated with increased grey matter volume in the frontal lobe, at the level of the right anterior 


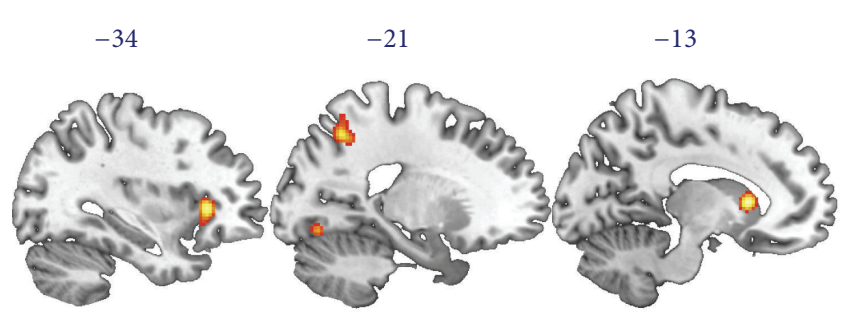

LH

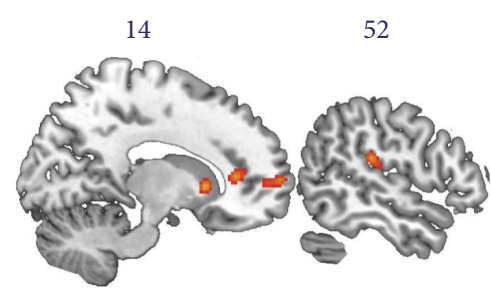

$\mathrm{RH}$

FIgURE 1: Results of ALE analysis on fMRI studies of meditation. The ALE map shows brain areas activated during meditation, encompassing bilaterally the caudate nuclei and insula, precuneus, middle and superior temporal gyrus, and precentral gyrus in the left hemisphere (LH) and the anterior cingulate cortex, superior frontal gyrus, parahippocampal gyrus, inferior parietal lobule, and middle occipital gyrus in the right hemisphere (RH).

cingulate cortex and left middle and medial frontal gyrus. We also found increased grey matter volume in meditators at the level of the left precuneus and fusiform gyrus and the right thalamus (Figure 3).

\section{Discussion}

The main aim of the present study was to identify the neural network activated during meditation and to explore structural and functional brain modifications in expert meditators. We also aimed to explore the relationship between meditation practice and the neural mechanisms that allow maintaining the positive effects of meditation training. For this purpose we adopted ALE analysis, a technique used widely in coordinatebased meta-analyses of neuroimaging data [58]. The results of this study shed light on the neural underpinnings of the positive effects of meditation practice and suggest the existence of a neural network responsible for these effects in meditators' everyday life.

The first question we tried to answer was which brain areas were activated during mediation. We used ALE analysis to identify the neural networks activated during meditation tasks and carried out the coordinate-based meta-analysis on experimental studies that required participants to meditate during the fMRI scan, regardless of their previous experience. We found that a set of brain areas spanning from the occipital to the frontal lobes was more highly activated during the meditation condition than during the control condition. This network included areas involved in processing self-relevant information, such as the precuneus [59], in processing selfregulation, focused problem-solving, and adaptive behavior, such as the anterior cingulate cortex [60], in interoception
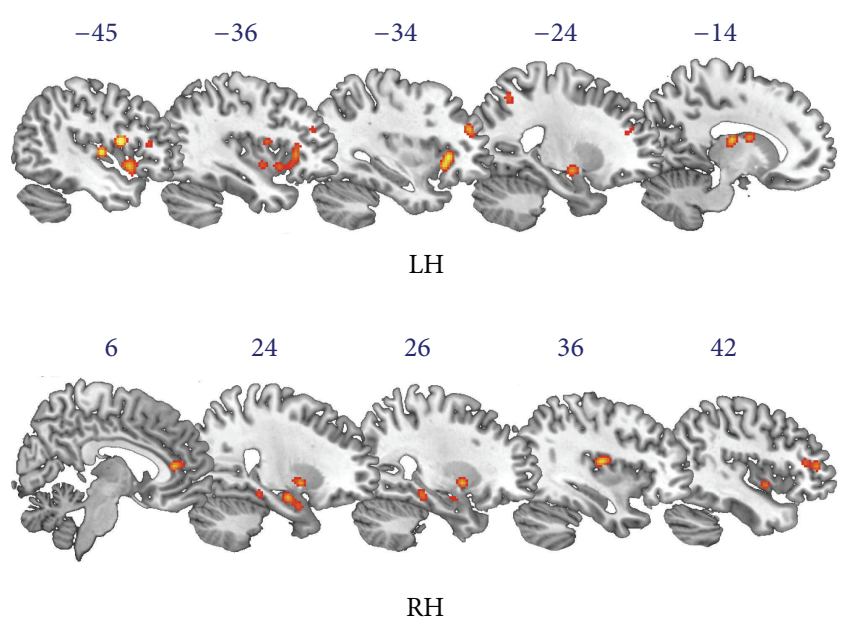

FIGURE 2: Results of ALE analysis on functional modifications in meditators. The ALE map shows brain areas that are more highly activated in meditators than controls. This network includes bilaterally the middle frontal gyrus, precentral gyrus, anterior cingulate cortex, insula, and claustrum. In the left hemisphere (LH) we found activation of the inferior frontal gyrus, precuneus, caudate nucleus, and thalamus, and in the right hemisphere (RH) we found activation in the medial frontal gyrus, parahippocampal gyrus, middle occipital gyrus, inferior parietal lobule, and lentiform nucleus.

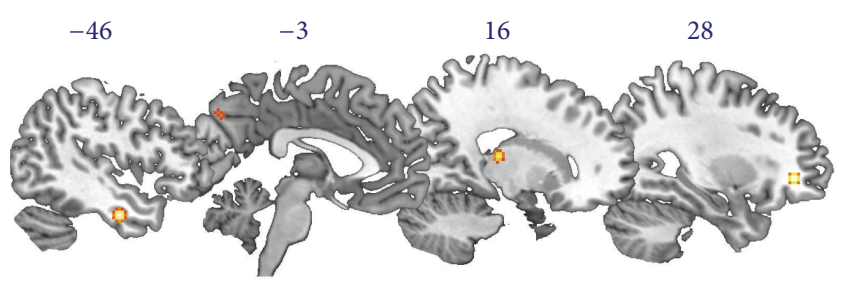

LH

RH

FIGURE 3: Results of the ALE analysis of structural modifications in meditators. The ALE map shows increased grey matter volume in meditators in the right hemisphere $(\mathrm{RH})$ at the level of the anterior cingulate cortex and thalamus and in the left hemisphere (LH) at the level of the middle and medial frontal gyrus, precuneus, and fusiform gyrus.

and in monitoring internal body states, such as the insula [61], in reorienting attention, such as the angular gyrus [62], and in processing the "experiential enactive self," such as the premotor cortex and superior frontal gyrus [63]. It is not surprising that meditation induces higher activation in all of these areas, because the mental state during meditation is mainly characterized by full attention to internal and external experiences as they occur in the present moment [15].

As previously described, meditation practice has been found to promote well-being by fostering cognitive and emotional functioning [6]. Indeed, the positive effects achieved during the training sessions were generalized to everyday life, enhancing both cognitive (i.e., memory, attention, problemsolving, and executive functions) and emotional (i.e., prosocial behavior) functioning in expert meditators. Using the 
ALE method, we tried to address the question about the brain underpinnings of pervasive positive effects of meditation in expert meditators' daily lives. We carried out an ALE analysis that included studies which compared activations in expert meditators and control participants in a wide range of cognitive and emotional domains (Table 1). Results of the ALE analysis showed that meditators, as compared with controls, showed greater activation in a wide network of areas encompassing bilaterally the frontal, parietal, and temporal regions. In addition to areas also activated during meditation (i.e., the middle occipital gyrus, inferior parietal lobule, precuneus, anterior cingulate cortex, precentral gyrus, insula, and caudate nuclei), this network of areas also included thebilateral middle frontal gyrus, inferior frontal gyrus, and thalamus in the left hemisphere and the medial frontal gyrus and lentiform nucleus in the right hemisphere. The network of areas we found more highly activated in expert meditators than in nonmeditators has recently been hypothesized to be part of the enactive experiential self network, which integrates efferent and reafferent processes concerning exteroception, proprioception, kinesthesia, and interoception [63]. Furthermore, it was previously thought that these areas were involved in self-referential processes [64-66], perspective taking [67], cognitive distancing [6871], and sustained attention [72]. In fact, they were found to be more highly activated in Buddhist meditators [1]. Expert meditators also showed higher activations in the parahippocampal cortex, which has repeatedly been found to be involved in memory formation and retrieval $[73,74]$ as well as in high-level perception, especially in perceiving complex and ambiguous visual stimuli $[75,76]$. The higher activation we found in expert meditators may account at least in part for enhanced attention, memory, and perceptual abilities reported in previous studies [15].

Results of the ALE analysis of sMRI studies showed increased grey matter volume in meditators compared to control groups in the right anterior cingulate cortex, left middle and medial frontal gyrus, left precuneus and fusiform gyrus, and right thalamus. It could be that the increased grey matter volume in the anterior cingulate cortex of meditators accounts for the improvement of specific abilities such as self-regulation, self-control, focused problem-solving, and adaptive behavioral responses under changing conditions [60], which are strictly associated with the functioning of the anterior cingulate cortex. Furthermore, the anterior cingulate cortex has recently been proposed to mediate the positive effects of meditation on prosocial behavior [63]. Nevertheless, it is difficult to state whether this difference as well as many other aspects of cognitive functioning is due to meditation practice or to previous individual predisposition. Studies comparing individuals before and after meditation training may help to clarify this point. Hölzel and colleagues [48] found increased grey matter concentration from preto post-MBSR training at the level of the temporoparietal junction, cerebellum, and posterior cingulate cortex. Furthermore, Kurth and colleagues [50] found a shifting in brain asymmetry at the level of the precuneus that was significantly correlated with number of years of practice. These results, taken together with results of the present ALE meta-analysis, which also found structural change in precuneus volume in meditators compared to controls, suggest that while structural differences at the level of the anterior cingulate cortex dispose to meditation, structural changes after meditation are strongly associated with changes in the posterior cingulate cortex and precuneus. The precuneus, which is located in the posteromedial portion of the parietal lobe, was recently found to be involved in a wide range of highly integrated tasks such as visuospatial imagery, episodic memory retrieval, and selfprocessing operations [59]. It shows widespread connectivity patterns with cortical and subcortical brain regions, such as the prefrontal cortex, anterior cingulate cortex, claustrum, caudate nucleus, and putamen [59]. The wide range of precuneus connections could account for its involvement in many high-level cognitive tasks. Specifically, involvement of the precuneus in self-referential processing could explain why it is so important in meditation practice. The precuneus was found to be involved in self-relevant information processing when self-relevant traits were compared with selfirrelevant traits [77]. It was also found to be involved during the performance of goal-directed actions when compared with passive stimulus viewing [78], the conscious resting state $[79,80]$, and the enhanced consciousness state of yoga meditation [55]. All of this evidence converges to suggest that the precuneus has a pivotal role in sustaining the positive effects of meditation practice especially because of its involvement in gathering self-relevant information and in representing the self and the external world [59].

Regarding the differences among meditation techniques, as reported above, meditation practices can be grossly divided into two different approaches: focused attention meditation and open monitoring meditation. Anyway, most meditation approaches use both types of practices complementarily $[3,4]$ and it has been proposed that all meditation techniques share a central process that supports their common goal, that is, inducing relaxation, regulating attention, and developing an attitude of detachment from one's own thoughts [57]. Our results strongly support the existence of a dedicate brain network that supports the general aspects of meditation effects. Actually, other than confirming the role of the caudate body, entorhinal cortex, and medial prefrontal cortex [57], the present study, using a large sample of experimental studies, sheds some light on other sets of brain areas which may be essential in supporting the general aspects of meditation effects.

\section{Conclusions}

Overall, results of the present ALE analysis suggest that meditation practice induces functional and structural brain modifications, especially in areas involved in self-referential processes, including self-awareness and self-regulation [63], as well as in areas involved in attention, executive functions, and memory formations [76]. Structural and functional modifications in this network may be the biological substrate of the pervasive effect of meditation practice in everyday life. These findings, taken together with previous ones, are leading to new applications of meditation practice in clinical populations and in disease prevention, especially in at-risk 
groups such as the elderly. In light of recent findings on the potential effect of meditation on age-related cognitive decline $[22,23]$, it could be intriguing to understand whether neurobiological changes promoted by meditation practice contribute to forming the so-called "Cognitive Reserve" [81]. Possible applications to a wide range of mental disorders affecting self-regulation and self-awareness, such as mood disorders [82, 83], anxiety disorders [84], and substance abuse [85], have also to be considered. In any case, further investigations comparing both psychological and neural effects of meditation practice are needed before any conclusions can be drawn.

\section{Conflict of Interests}

The authors declare that there is no conflict of interests regarding the publication of this paper.

\section{References}

[1] B. Tomasino, A. Chiesa, and F. Fabbro, "Disentangling the neural mechanisms involved in Hinduism- and Buddhism-related meditations," Brain and Cognition, vol. 90, pp. 32-40, 2014.

[2] R. J. Davidson and D. J. Goleman, "The role of attention in meditation and hypnosis: a psychobiological perspective on transformations of consciousness," International Journal of Clinical and Experimental Hypnosis, vol. 25, no. 4, pp. 291-308, 1977.

[3] J. Andresen, "Meditation meets behavioural medicine: the story of experimental research on meditation," Journal of Consciousness Studies, vol. 7, no. 11-12, pp. 17-73, 2000.

[4] B. A. Wallace, "The Buddhist tradition of Samatha: methods for refining and examining consciousness," Journal of Consciousness Studies, vol. 6, no. 3, pp. 175-187, 1999.

[5] K. W. Brown and R. M. Ryan, "The benefits of being present: mindfulness and its role in psychological well-being," Journal of Personality and Social Psychology, vol. 84, no. 4, pp. 822-848, 2003.

[6] E. Luders, A. W. Toga, N. Lepore, and C. Gaser, "The underlying anatomical correlates of long-term meditation: larger hippocampal and frontal volumes of gray matter," NeuroImage, vol. 45 , no. 3, pp. 672-678, 2009.

[7] A. P. Jha, E. A. Stanley, A. Kiyonaga, L. Wong, and L. Gelfand, "Examining the protective effects of mindfulness training on working memory capacity and affective experience," Emotion, vol. 10, no. 1, pp. 54-64, 2010.

[8] A. P. Jha, J. Krompinger, and M. J. Baime, "Mindfulness training modifies subsystems of attention," Cognitive, Affective and Behavioral Neuroscience, vol. 7, no. 2, pp. 109-119, 2007.

[9] N. Srinivasan and S. Baijal, "Concentrative meditation enhances preattentive processing: a mismatch negativity study," NeuroReport, vol. 18, no. 16, pp. 1709-1712, 2007.

[10] K. A. MacLean, E. Ferrer, S. R. Aichele et al., "Intensive meditation training improves perceptual discrimination and sustained attention," Psychological Science, vol. 21, no. 6, pp. 829-839, 2010.

[11] P. Condon, G. Desbordes, W. B. Miller, and D. DeSteno, "Meditation increases compassionate responses to suffering," Psychological Science, vol. 24, no. 10, pp. 2125-2127, 2013.

[12] B. K. Hölzel, U. Ott, H. Hempel et al., "Differential engagement of anterior cingulate and adjacent medial frontal cortex in adept meditators and non-meditators," Neuroscience Letters, vol. 421, no. 1, pp. 16-21, 2007.

[13] A. Lutz, J. Dunne, and R. Davidson, "Meditation and the neuroscience of consciousness: an introduction," in The Cambridge Handbook of Consciousness, P. Zelazo, M. Moscovitch, and E. Thompson, Eds., pp. 499-554, Cambridge University Press, Cambridge, UK, 2007.

[14] A. Lutz, H. A. Slagter, J. D. Dunne, and R. J. Davidson, "Attention regulation and monitoring in meditation," Trends in Cognitive Sciences, vol. 12, no. 4, pp. 163-169, 2008.

[15] A. Chiesa, R. Calati, and A. Serretti, "Does mindfulness training improve cognitive abilities? A systematic review of neuropsychological findings," Clinical Psychology Review, vol. 31, no. 3, pp. 449-464, 2011.

[16] R. Chambers, B. C. Y. Lo, and N. B. Allen, "The impact of intensive mindfulness training on attentional control, cognitive style, and affect," Cognitive Therapy and Research, vol. 32, no. 3, pp. 303-322, 2008.

[17] H. A. Slagter, A. Lutz, L. L. Greischar et al., "Mental training affects distribution of limited brain resources," PLoS Biology, vol. 5, no. 6, article el38, 2007.

[18] F. Zeidan, S. K. Johnson, B. J. Diamond, Z. David, and P. Goolkasian, "Mindfulness meditation improves cognition: evidence of brief mental training," Consciousness and Cognition, vol. 19, no. 2, pp. 597-605, 2010.

[19] E. Hargus, C. Crane, T. Barnhofer, and J. M. G. Williams, "Effects of mindfulness on meta-awareness and specificity of describing prodromal symptoms in suicidal depression," Emotion, vol. 10, no. 1, pp. 34-42, 2010.

[20] A. Heeren, N. van Broeck, and P. Philippot, "The effects of mindfulness on executive processes and autobiographical memory specificity," Behaviour Research and Therapy, vol. 47, no. 5, pp. 403-409, 2009.

[21] C. N. M. Ortner, S. J. Kilner, and P. D. Zelazo, “Mindfulness meditation and reduced emotional interference on a cognitive task," Motivation and Emotion, vol. 31, no. 4, pp. 271-283, 2007.

[22] T. Gard, B. K. Holzel, and S. W. Lazar, "The potential effects of meditation on age-related cognitive decline: a systematic review," Annals of the New York Academy of Sciences, vol. 1307, no. 1, pp. 89-103, 2014.

[23] E. Larouche, C. Hudon, and S. Goulet, "Potential benefits of mindfulness-based interventions in mild cognitive impairment and Alzheimer's disease: an interdisciplinary perspective," Behavioural Brain Research, vol. 276, pp. 199-212, 2015.

[24] E. Luders, F. Kurth, A. W. Toga, K. L. Narr, and C. Gaser, "Meditation effects within the hippocampal complex revealed by voxel-based morphometry and cytoarchitectonic probabilistic mapping," Frontiers in Psychology, vol. 4, article 398, 2013.

[25] C. D. Conrad, K. J. McLaughlin, J. S. Harman et al., "Chronic glucocorticoids increase hippocampal vulnerability to neurotoxicity under conditions that produce CA3 dendritic retraction but fail to impair spatial recognition memory," Journal of Neuroscience, vol. 27, no. 31, pp. 8278-8285, 2007.

[26] R. A. Baer, "Mindfulness training as a clinical intervention: a conceptual and empirical review," Clinical Psychology: Science and Practice, vol. 10, no. 2, pp. 125-143, 2003.

[27] P. Grossman, L. Niemann, S. Schmidt, and H. Walach, "Mindfulness-based stress reduction and health benefits: a metaanalysis," Journal of Psychosomatic Research, vol. 57, no. 1, pp. 35-43, 2004. 
[28] A. P. King, T. M. Erickson, N. D. Giardino et al., "A pilot study of group mindfulness-based cognitive therapy (MBCT) for combat veterans with posttraumatic stress disorder (PTSD)," Depression and Anxiety, vol. 30, no. 7, pp. 638-645, 2013.

[29] S. H. Ma and J. D. Teasdale, "Mindfulness-based cognitive therapy for depression: replication and exploration of differential relapse prevention effects," Journal of Consulting and Clinical Psychology, vol. 72, no. 1, pp. 31-40, 2004.

[30] J. D. Teasdale, Z. V. Segal, J. M. G. Williams, V. A. Ridgewaya, J. M. Soulsby, and M. A. Lau, "Prevention of relapse/recurrence in major depression by mindfulness-based cognitive therapy," Journal of Consulting and Clinical Psychology, vol. 68, no. 4, pp. 615-623, 2000.

[31] W. Kuyken, S. Byford, R. S. Taylor et al., "Mindfulness-based cognitive therapy to prevent relapse in recurrent depression," Journal of Consulting and Clinical Psychology, vol. 76, no. 6, pp. 966-978, 2008.

[32] J. A. Brewer and K. A. Garrison, "The posterior cingulate cortex as a plausible mechanistic target of meditation: findings from neuroimaging," Annals of the New York Academy of Sciences, vol. 1307, no. 1, pp. 19-27, 2014.

[33] J. Xu, A. Vik, I. R. Groote et al., "Nondirective meditation activates default mode network and areas associated with memory retrieval and emotional processing," Frontiers in Human Neuroscience, vol. 8, article 86, 2014.

[34] S. Davanger, Ø. Ellingsen, A. Holen, and K. Hugdahl, "Meditation-specific prefrontal cortical activation during Acem meditation: an fMRI study," Perceptual and Motor Skills, vol. 111, no. 1, pp. 291-306, 2010.

[35] J. Dickenson, E. T. Berkman, J. Arch, and M. D. Lieberman, "Neural correlates of focused attention during a brief mindfulness induction," Social Cognitive and Affective Neuroscience, vol. 8, no. 1, Article ID nss030, pp. 40-47, 2013.

[36] M. Engström, J. Pihlsgård, P. Lundberg, and B. Söderfeldt, "Functional magnetic resonance imaging of hippocampal activation during silent mantra meditation," The Journal of Alternative and Complementary Medicine, vol. 16, no. 12, pp. 1253-1258, 2010.

[37] M. Allen, M. Dietz, K. S. Blair et al., "Cognitive-affective neural plasticity following active-controlled mindfulness intervention," Journal of Neuroscience, vol. 32, no. 44, pp. 1560115610, 2012.

[38] J. A. Grant, J. Courtemanche, and P. Rainville, "A non-elaborative mental stance and decoupling of executive and pain-related cortices predicts low pain sensitivity in Zen meditators," Pain, vol. 152, no. 1, pp. 150-156, 2011.

[39] A. Lutz, D. R. McFarlin, D. M. Perlman, T. V. Salomons, and R. J. Davidson, "Altered anterior insula activation during anticipation and experience of painful stimuli in expert meditators," NeuroImage, vol. 64, no. 1, pp. 538-546, 2013.

[40] J. S. Mascaro, J. K. Rilling, L. T. Negi, and C. L. Raison, "Pre-existing brain function predicts subsequent practice of mindfulness and compassion meditation," NeuroImage, vol. 69, pp. 35-42, 2013.

[41] J. S. Mascaro, J. K. Rilling, L. Tenzin Negi, and C. L. Raison, "Compassion meditation enhances empathic accuracy and related neural activity," Social Cognitive and Affective Neuroscience, vol. 8, no. 1, Article ID nss095, pp. 48-55, 2013.

[42] N. A. S. Farb, Z. V. Segal, H. Mayberg et al., "Attending to the present: Mindfulness meditation reveals distinct neural modes of self-reference," Social Cognitive and Affective Neuroscience, vol. 2, no. 4, pp. 313-322, 2007.
[43] W. Hasenkamp, C. D. Wilson-Mendenhall, E. Duncan, and L. W. Barsalou, "Mind wandering and attention during focused meditation: a fine-grained temporal analysis of fluctuating cognitive states," NeuroImage, vol. 59, no. 1, pp. 750-760, 2012.

[44] J. A. Brefczynski-Lewis, A. Lutz, H. S. Schaefer, D. B. Levinson, and R. J. Davidson, "Neural correlates of attentional expertise in long-term meditation practitioners," Proceedings of the National Academy of Sciences of the United States of America, vol. 104, no. 27, pp. 11483-11488, 2007.

[45] V. A. Taylor, J. Grant, V. Daneault et al., "Impact of mindfulness on the neural responses to emotional pictures in experienced and beginner meditators," NeuroImage, vol. 57, no. 4, pp. 15241533, 2011.

[46] Y.-Y. Tang, R. Tang, and M. I. Posner, "Brief meditation training induces smoking reduction," Proceedings of the National Academy of Sciences of the United States of America, vol. 110, no. 34, pp. 13971-13975, 2013.

[47] A. Guleria, U. Kumar, S. S. K. Kishan, and C. L. Khetrapal, "Effect of "SOHAM" meditation on the human brain: an fMRI study," Psychiatry Research-Neuroimaging, vol. 214, no. 3, pp. 462-465, 2013.

[48] B. K. Hölzel, J. Carmody, M. Vangel et al., "Mindfulness practice leads to increases in regional brain gray matter density," Psychiatry Research-Neuroimaging, vol. 191, no. 1, pp. 36-43, 2011.

[49] D.-H. Kang, H. J. Jo, W. H. Jung et al., “The effect of meditation on brain structure: cortical thickness mapping and diffusion tensor imaging," Social Cognitive and Affective Neuroscience, vol. 8, no. 1, Article ID nss056, pp. 27-33, 2013.

[50] F. Kurth, A. Mackenzie-Graham, A. W. Toga, and E. Luders, "Shifting brain asymmetry: the link between meditation and structural lateralization," Social Cognitive and Affective Neuroscience, vol. 8, pp. 27-33, 2014.

[51] M.-K. Leung, C. C. H. Chan, J. Yin, C.-F. Lee, K.-F. So, and T. M. C. Lee, "Increased gray matter volume in the right angular and posterior parahippocampal gyri in loving-kindness meditators," Social Cognitive \& Affective Neuroscience, vol. 8, no. 1, pp. 34-39, 2013.

[52] E. Luders, A. W. Toga, N. Lepore, and C. Gaser, "The underlying anatomical correlates of long-term meditation: larger hippocampal and frontal volumes of gray matter," NeuroImage, vol. 45 , no. 3 , pp. $672-678,2009$.

[53] P. Vestergaard-Poulsen, M. Van Beek, J. Skewes et al., "Longterm meditation is associated with increased gray matter density in the brain stem," NeuroReport, vol. 20, no. 2, pp. 170-174, 2009.

[54] G.-X. Wei, T. Xu, F.-M. Fan et al., "Can taichi reshape the brain? A brain morphometry study," PLoS ONE, vol. 8, no. 4, Article ID e61038, 2013.

[55] H. C. Lou, T. W. Kjaer, L. Friberg, G. Wildschiodtz, S. Holm, and M. Nowak, "A ${ }^{15} \mathrm{O}-\mathrm{H}_{2} \mathrm{O}$ PET study of meditation and the resting state of normal consciousness," Human Brain Mapping, vol. 7, no. 2, pp. 98-105, 1999.

[56] A. Manna, A. Raffone, M. G. Perrucci et al., "Neural correlates of focused attention and cognitive monitoring in meditation," Brain Research Bulletin, vol. 82, no. 1-2, pp. 46-56, 2010.

[57] M. Sperduti, P. Martinelli, and P. Piolino, "A neurocognitive model of meditation based on activation likelihood estimation (ALE) meta-analysis," Consciousness and Cognition, vol. 21, no. 1, pp. 269-276, 2012.

[58] S. B. Eickhoff, A. R. Laird, C. Grefkes, L. E. Wang, K. Zilles, and P. T. Fox, "Coordinate-based activation likelihood estimation 
meta-analysis of neuroimaging data: a random-effects approach based on empirical estimates of spatial uncertainty," Human Brain Mapping, vol. 30, no. 9, pp. 2907-2926, 2009.

[59] A. E. Cavanna and M. R. Trimble, "The precuneus: a review of its functional anatomy and behavioural correlates," Brain, vol. 129, no. 3, pp. 564-583, 2006.

[60] K. C. R. Fox, S. Nijeboer, M. L. Dixon et al., "Is meditation associated with altered brain structure? A systematic review and meta-analysis of morphometric neuroimaging in meditation practitioners," Neuroscience \& Biobehavioral Reviews, vol. 43, pp. 48-73, 2014.

[61] A. D. Craig, "How do you feel? Interoception: the sense of the physiological condition of the body," Nature Reviews Neuroscience, vol. 3, no. 8, pp. 655-666, 2002.

[62] M. L. Seghier, "The angular gyrus: multiple functions and multiple subdivisions," Neuroscientist, vol. 19, no. 1, pp. 43-61, 2013.

[63] D. R. Vago and S. A. David, "Self-awareness, self-regulation, and self-transcendence (S-ART): a framework for understanding the neurobiological mechanisms of mindfulness," Frontiers in Human Neuroscience, vol. 6, no. 296, pp. 1-30, 2012.

[64] G. Northoff, A. Heinzel, M. de Greck, F. Bermpohl, H. Dobrowolny, and J. Panksepp, "Self-referential processing in our brain - a meta-analysis of imaging studies on the self," Neuroimage, vol. 31, no. 1, pp. 440-457, 2006.

[65] L. van der Meer, S. Costafreda, A. Aleman, and A. S. David, "Self-reflection and the brain: a theoretical review and meta-analysis of neuroimaging studies with implications for schizophrenia," Neuroscience and Biobehavioral Reviews, vol. 34, no. 6, pp. 935-946, 2010.

[66] S. Whitfield-Gabrieli, J. M. Moran, A. Nieto-Castañón, C. Triantafyllou, R. Saxe, and J. D. E. Gabrieli, "Associations and dissociations between default and self-reference networks in the human brain," NeuroImage, vol. 55, no. 1, pp. 225-232, 2011.

[67] N. David, B. H. Bewernick, M. X. Cohen et al., "Neural representations of self versus other: visual-spatial perspective taking and agency in a virtual ball-tossing game," Journal of Cognitive Neuroscience, vol. 18, no. 6, pp. 898-910, 2006.

[68] M. Beauregard, J. Lévesque, and P. Bourgouin, "Neural correlates of conscious self-regulation of emotion," The Journal of Neuroscience, vol. 21, no. 18, Article ID RC165, 2001.

[69] C. S. Carter, M. Mintun, and J. D. Cohen, "Interference and facilitation effects during selective attention: an $\mathrm{H}_{2}{ }^{15} \mathrm{O}$ PET study of stroop task performance," NeuroImage, vol. 2, no. 4, pp. 264-272, 1995.

[70] J. Levesque, F. Eugène, Y. Joanette et al., "Neural circuitry underlying voluntary suppression of sadness," Biological Psychiatry, vol. 53, no. 6, pp. 502-510, 2003.

[71] A. W. MacDonald III, J. D. Cohen, V. A. Stenger, and C. S. Carter, "Dissociating the role of the dorsolateral prefrontal and anterior cingulate cortex in cognitive control," Science, vol. 288, no. 5472, pp. 1835-1838, 2000.

[72] M. I. Posner and S. E. Petersen, "The attention system of the human brain," Annual Review of Neuroscience, vol. 13, pp. 2542, 1990.

[73] W. B. Scoville and B. Milner, "Loss of recent memory after bilateral hippocampal lesions," Journal of Neurology, Neurosurgery \& Psychiatry, vol. 20, no. 1, pp. 11-21, 1957.

[74] L. R. Squire and S. Zola-Morgan, "The medial temporal lobe memory system,” Science, vol. 253, no. 5026, pp. 1380-1386, 1991.
[75] M. D. Barense, R. N. A. Henson, A. C. H. Lee, and K. S. Graham, "Medial temporal lobe activity during complex discrimination of faces, objects, and scenes: effects of viewpoint," Hippocampus, vol. 20, no. 3, pp. 389-401, 2010.

[76] R. K. Lech and B. Suchan, "The medial temporal lobe: memory and beyond," Behavioural Brain Research, vol. 254, pp. 45-49, 2013.

[77] T. Kircher, V. Arolt, A. Jansen et al., "Effect of cognitivebehavioral therapy on neural correlates of fear conditioning in panic disorder," Biological Psychiatry, vol. 73, no. 1, pp. 93-101, 2013.

[78] G. L. Shulman, J. A. Fiez, M. Corbetta et al., "Common blood flow changes across visual tasks: II. Decreases in cerebral cortex," Journal of Cognitive Neuroscience, vol. 9, no. 5, pp. 648663, 1997.

[79] R. C. Gur, L. H. Mozley, P. D. Mozley et al., "Sex differences in regional cerebral glucose metabolism during a resting state," Science, vol. 267, no. 5197, pp. 528-531, 1995.

[80] J. R. Binder, J. A. Frost, T. A. Hammeke, P. S. F. Bellgowan, S. M. Rao, and R. W. Cox, "Conceptual processing during the conscious resting state: a functional MRI study," Journal of Cognitive Neuroscience, vol. 11, no. 1, pp. 80-95, 1999.

[81] C. Solé-Padullés, D. Bartrés-Faz, C. Junqué et al., "Brain structure and function related to cognitive reserve variables in normal aging, mild cognitive impairment and Alzheimer's disease," Neurobiology of Aging, vol. 30, no. 7, pp. 1114-1124, 2009.

[82] F. M. Howells, V. L. Ives-Deliperi, N. R. Horn, and D. J. Stein, "Mindfulness based cognitive therapy improves frontal control in bipolar disorder: a pilot EEG study," BMC Psychiatry, vol. 12, article 15, 2012.

[83] H. F. Coelho, P. H. Canter, and E. Ernst, "Mindfulness-based cognitive therapy: evaluating current evidence and informing future research," Journal of Consulting and Clinical Psychology, vol. 75, no. 6, pp. 1000-1005, 2007.

[84] Y. W. Kim, S.-H. Lee, T. K. Choi et al., "Effectiveness of mindfulness-based cognitive therapy as an adjuvant to pharmacotherapy in patients with panic disorder or generalized anxiety disorder," Depression \& Anxiety, vol. 26, no. 7, pp. 601-606, 2009.

[85] S. Bowen, K. Witkiewitz, T. M. Dillworth et al., "Mindfulness meditation and substance use in an incarcerated population," Psychology of Addictive Behaviors, vol. 20, no. 3, pp. 343-347, 2006.

[86] J. D. Creswell, B. M. Way, N. I. Eisenberger, and M. D. Lieberman, "Neural correlates of dispositional mindfulness during affect labeling," Psychosomatic Medicine, vol. 69, no. 6, pp. 560-565, 2007.

[87] X. Ding, Y. Y. Tang, C. Cao et al., "Short-term meditation modulates brain activity of insight evoked with solution cue," Social Cognitive and Affective Neuroscience, 2014.

[88] N. A. S. Farb, A. K. Anderson, H. Mayberg, J. Bean, D. McKeon, and Z. V. Segal, "Minding one's emotions: mindfulness training alters the neural expression of sadness," Emotion, vol. 10, no. 1, pp. 25-33, 2010.

[89] N. A. S. Farb, Z. V. Segal, and A. K. Anderson, "Mindfulness meditation training alters cortical representations of interoceptive attention," Social Cognitive and Affective Neuroscience, vol. 8, no. 1, Article ID nss066, pp. 15-26, 2013.

[90] V. L. Ives-Deliperi, M. Solms, and E. M. Meintjes, “The neural substrates of mindfulness: an fMRI investigation," Social Neuroscience, vol. 6, no. 3, pp. 231-242, 2011. 
[91] J. H. Jang, W. H. Jung, D. H. Kang et al., "Increased default mode network connectivity associated with meditation," Neuroscience Letters, vol. 487, no. 3, pp. 358-362, 2011.

[92] L. A. Kilpatrick, B. Y. Suyenobu, S. R. Smith et al., "Impact of mindfulness-based stress reduction training on intrinsic brain connectivity," NeuroImage, vol. 56, no. 1, pp. 290-298, 2011.

[93] T. M. C. Lee, M.-K. Leung, W.-K. Hou et al., "Distinct neural activity associated with focused-attention meditation and loving-kindness meditation," PLoS ONE, vol. 7, no. 8, Article ID e40054, 2012.

[94] A. Lutz, L. L. Greischar, D. M. Perlman, and R. J. Davidson, "BOLD signal in insula is differentially related to cardiac function during compassion meditation in experts vs. novices," NeuroImage, vol. 47, no. 3, pp. 1038-1046, 2009.

[95] D. A. Monti, K. M. Kash, E. J. S. Kunkel et al., "Changes in cerebral blood flow and anxiety associated with an 8-week mindfulness programme in women with breast cancer," Stress and Health, vol. 28, no. 5, pp. 397-407, 2012.

[96] D. W. Orme-Johnson, R. H. Schneider, Y. D. Son, S. Nidich, and Z.-H. Cho, "Neuroimaging of meditation's effect on brain reactivity to pain," NeuroReport, vol. 17, no. 12, pp. 1359-1363, 2006.

[97] D. J. J. Wang, H. Rao, M. Korczykowski et al., "Cerebral blood flow changes associated with different meditation practices and perceived depth of meditation," Psychiatry ResearchNeuroimaging, vol. 191, no. 1, pp. 60-67, 2011. 

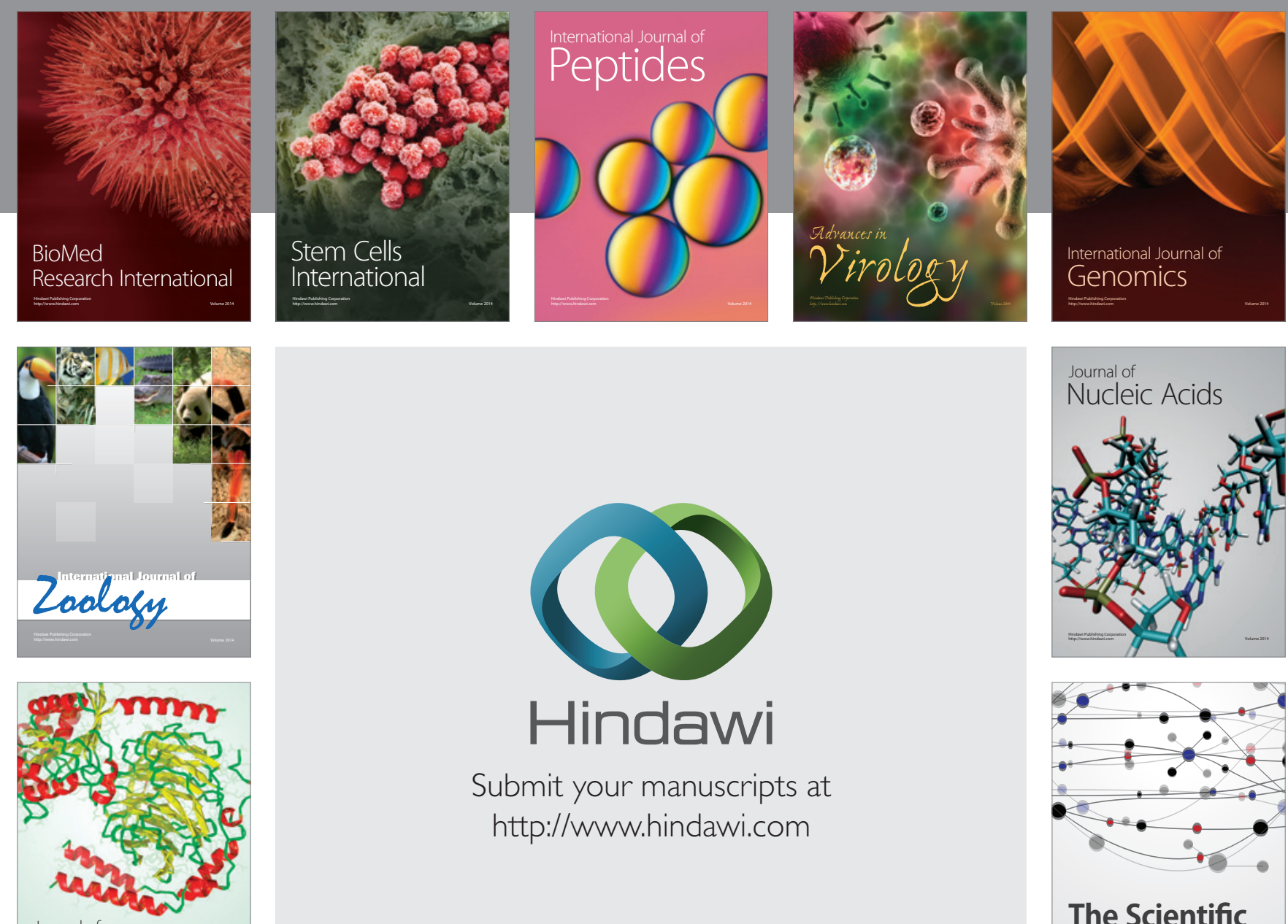

Submit your manuscripts at

http://www.hindawi.com

Journal of
Signal Transduction
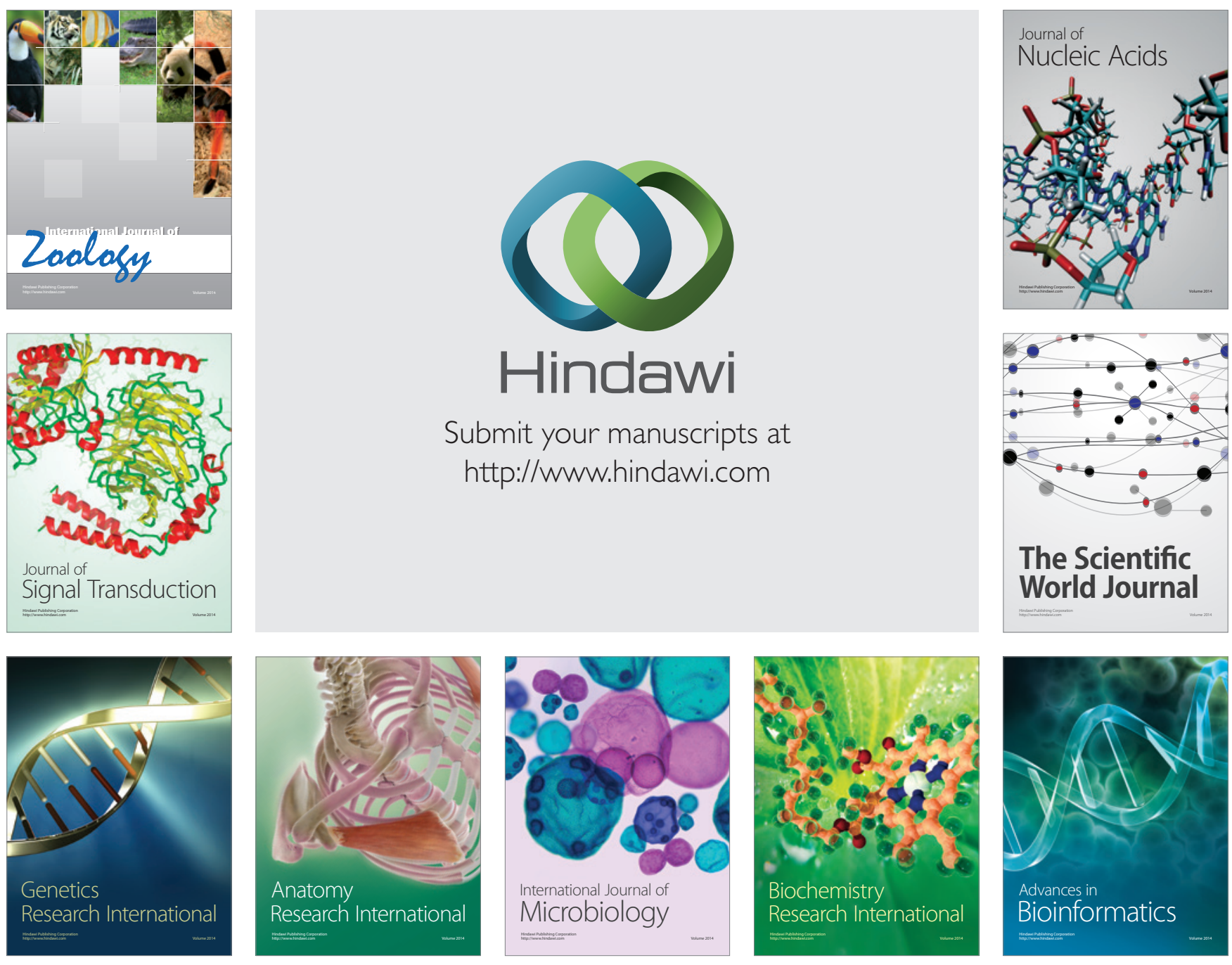

The Scientific World Journal
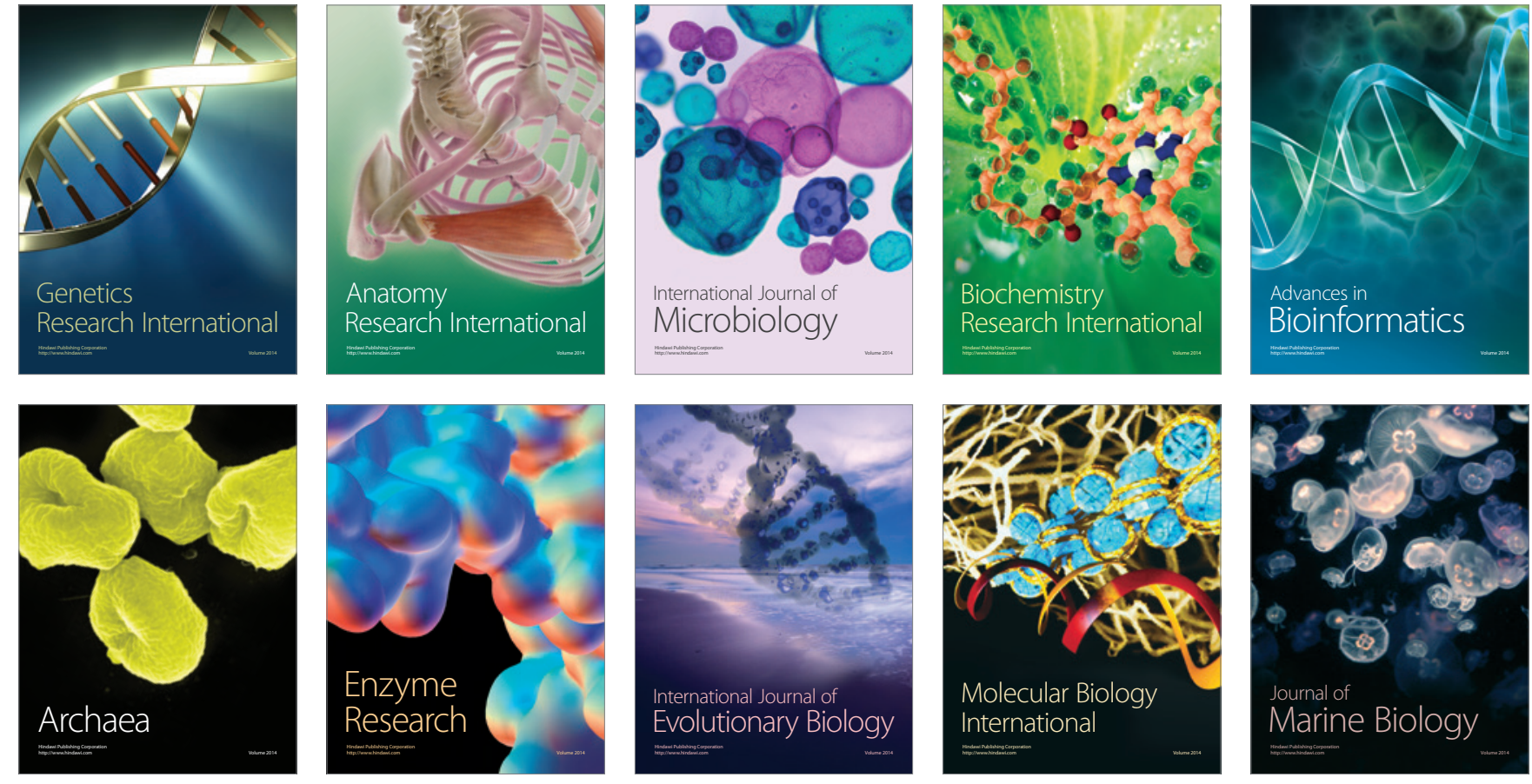